\title{
Variation of antibody responses to Plasmodium falciparum MSP1- 19 antigen with parasitaemia and IL4vntr polymorphism in Khartoum state, Sudan
}

\author{
Hind M. Abushama ${ }^{1}$ - Inas A. AbdelRahman ${ }^{1} \cdot$ Hiba Ali $^{1} \cdot$ Tasneem Mowia $^{1} \cdot$ Faisal Mousa $^{1}$. \\ Muzamil M. Abdelhamid ${ }^{2}$ Ibrahim M. ElHassan ${ }^{2}$
}

Received: 26 April 2020/Accepted: 3 November 2020/Published online: 17 November 2020

(C) Indian Society for Parasitology 2020

\begin{abstract}
A hospital-based cross-sectional study was conducted at Khartoum state to investigate the variation of antibody responses to Plasmodium falciparum 19-kDa C-terminal region of merozoite surface protein 1 antigen and the variation of human ILA polymorphism with parasitaemia. Measurements of natural acquisition of antiPlasmodium falciparum MSP1-19 IgG, IgG1 and IgG3 antibodies were performed using ELISA. Molecular characterization of ILAvntr polymorphism was achieved. We were able to detect a statistically significant negative correlation between parasitaemia and different age groups $(\mathrm{r}=-0.262$ and $p$ value $=0.043)$ and with anti-P.fMSP119 IgG1 $(\mathrm{r}=-0.418, p$ value $=0.047)$. Anti-P.fMSP1-19 IgG showed a significant difference among age groups $(p<0.001)$. Only anti-P.fMSPI-19 IgG showed a
\end{abstract}

Hind M. Abushama

hindabushama@hotmail.com

Inas A. AbdelRahman

enasdaff@hotmail.com

Hiba Ali

hibaali3010@hotmail.com

Tasneem Mowia

tasneemmoawia@hotmail.com

Faisal Mousa

faisalmousa86@gmail.com

Muzamil M. Abdelhamid

mahdi@iend.org

Ibrahim M. ElHassan

ibrahimelhassan@iend.org

1 Department of Zoology, Faculty of Science, University of Khartoum, Khartoum, Sudan

2 Institute of Endemic Diseases, University of Khartoum, Khartoum, Sudan significant association with general appearance $(p$ value $<0.001)$. The mean for total anti-P.fMSP1-19 IgG3 was statistically significantly higher in females compared to males $(p$ value $<0.001)$. There was no significant difference in the distribution of human IL4 vntr genotypic and allelic frequencies between cases and control group as well as among different clinical manifestation.We concluded that IgG1 levels to MSP1-19 were found to be negatively correlated with parasitaemia and anti-PfMSP $1-19 \operatorname{IgG}$ was significantly increased in ill and severely ill with age considered as a cofactor. Further studies are needed to ascertain the role of $\operatorname{IgG}$ and $\operatorname{IgG} 1$ in protection and to investigate the $\operatorname{IgG}$ and subclasses' response against other antigenic markers. These findings are valuable for advancing vaccine development by providing evidence supporting merozoite antigens as targets of protective immunity in humans.

Keywords Plasmodium falciparum · MSP1-19 . $\mathrm{IgG} \cdot \mathrm{IgG1} \cdot \mathrm{IgG} 3 \cdot$ IL4vntr $\cdot$ Parasitaemia $\cdot$ Sudan

\section{Background}

A substantial decline of Plasmodium falciparum infection has been witnessed in Sudan in the past few years following the implementation of new control strategies, even though malaria is still considered as the major cause of febrile illness in the country. Symptomatic malaria accounts for $20-40 \%$ of outpatient clinic visits and approximately $30 \%$ of hospital admissions. More than hundred thousand severe malaria cases being reported annually. Around one-third of malaria deaths is occurring among children below 5 years of age (Federal Ministry of Health 2016). Generally, the parasite species with the 
perennial transmission is Plasmodium falciparum ( $P$. falciparum), which is responsible for about $90 \%$ of reported malaria. In Sudan five malaria strata can be identified; Riverine, seasonal, high perennial, urban, and irrigated areas malaria (Malaria Control Programme 2008). Despite, the efforts of malaria control and eradication programs, most malaria cases in 2017 were in the WHO African Region (200 million or 92\%). This complicated situation is due mainly to the spread of drug resistance and insecticide resistance (World Malaria Report 2018). Immunity to malaria is complex partly due to the complicated life cycle of the parasite with different antigens expressed at different times and to the variation in host immune response against infection. However, It becomes clear that the development of immune protection against malaria includes both specific and non specific mechanism and that both cellular and humoral immune responses are involved (Marsh 1992; Perlmann and Troye-Blomberg 2002) with a possible role of $\mathrm{IgG}$ in protection as indicated in previous studies (Giha et al. 2010; Iriemenam et al. 2009; Adamou et al. 2019; Dobaño et al. 2019).

MSP-1 is the major surface antigen of merozoite and is the best-studied protein (Dodoo et al. 1999). Most of the studies on the MSP1 antibodies have been carried out in areas of stable hyper-holoendemic malaria, while only limited information is available from areas of unstable malaria with lower endemicity. In previous studies, $P$. falciparum MSP1-19 has been used to determine the specificity and longitudinal patterns of antibody responses and clinical protection against malaria (Nguer et al. 1997; A-Elgadir et al. 2008). The prevalence of MSP1 antibodies was associated with the clinical outcome of malaria infection, and it was age-dependent (A-Elgadir et al. 2008). A systematic review with meta-analysis of prospective cohort studies has shown a significant association between anti-merozoite IgG responses and incidence of $P$. falciparum malaria. The largest effect was observed with IgG to MSP-3 C terminus and MSP1-19 (Fowkes et al. 2010) with a significant variation in antibody response among individuals suffer from severe malaria (A-Elgadir et al. 2008). Moreover, IL-4, IL-10 and IL-13 gene polymorphisms were found to be related to the regulation of IgG responses to a number of antigens including MSP-1 (Lokossou et al. 2013). Measurement of serum anti-malarial antibodies is a useful marker of malaria exposure and might help in the classification of clinical disease outcome. Moreover, the certainty of finding a protective role of acquired immunity in malaria is the major drive for malaria vaccine development. Molecular characterization of infectious diseases with integrated analysis of variable phenotypic (parasitaemia, immunologic response) might help in the identification of susceptibility/resistance genes with an estimation of those genetically identically predisposed to infection. Besides, the identification of host genes (e.g. ILA genes) involved in the control of human infectious diseases might help in the identification of effective immune response in course of infection and further provide new approaches to modify the existent measurements used in the prevention and treatment of infectious diseases and probably in developing an effective vaccine. We aimed in this study to investigate the correlation of parasitological and immunological phenotypes of $P$. falciparum using the ILA gene, parasitaemia and anti-P.falciparumMSP1-19 $\mathrm{IgG}, \mathrm{IgG} 1$ and $\mathrm{IgG} 3$ as markers in Khartoum.

\section{Methods}

The current study is a cross sectional hospital-based study conducted at two hospitals in Khartoum state based on the high number of malaria cases presented to these hospitals (Federal Ministry of Health 2016). Ethical clearance for the study was obtained from the Sudanese Ministry of Health Ethical committee.

\section{Sample and questionnaire data collection}

The participants were included in this study after they presented to the hospital laboratory for symptoms commonly attributed to malaria and physically investigated by the hospital physician. Healthy controls were also selected from individuals admitted to the hospital and found to be free from malaria and were living in the same environmental setting as the study participants. Informed consent was obtained from all study participants. A questionnaire has been filled for each participant for parameters related to personal data, clinical symptoms, type of treatment, parasitological data and infections with other diseases. Clinical data related to malaria symptoms were recorded for all participants in the study as assessed by a physician in the hospital. Based on WHO criteria (Impaired consciousness, Respiratory distress (acidotic breathing) Multiple convulsions, Prostration, Shock, Pulmonary oedema (radiological), Abnormal bleeding, Jaundice), the general clinical appearance of the study participants was categorized into ill, severely ill and well.

A total of one hundred and twenty blood samples were collected from the study participants and controls. Duplicate thin and thick blood smears were prepared from finger prick by the laboratory technician. Plasmodium falciparium positive and negative samples were reinvestigated at the Department of Zoology lab, Khartoum University by microscopic diagnosis according to the WHO criteria.

Filter paper Whatman No 3 was used to collect blood samples from a finger prick for subsequent DNA extraction, PCR analysis and antibodies measurements. 
Duplicated labeled filter paper was used for each participant. Each filter paper was placed into plastic bags and preserved dry in the fridge at $4{ }^{\circ} \mathrm{C}$.

\section{Detection and identification of Plasmodium species (P.falciparum)}

The thick blood film was used for the detection of the malaria parasite and parasite count, while thin blood film was used for confirmation of species using microscopy (Gilles 1993).

DNA was extracted from the filter paper blood samples using the chelex extraction method (Plowe et al. 1997). This DNA was used to detect the parasite species and further to identify human ILA polymorphism.

Identification of plasmodium species using nested PCR was carried out using species-specific primers rPLU2, rPLU5, rFAL1 and rFAL2 to detect and identify the Plasmodium species (Snounou et al. 1993) as illustrated in Table 1.

The total volume of the reaction was $20 \mu \mathrm{l}$. The PCR reaction was carried out in $50 \mathrm{mM} \mathrm{KCl}, 10 \mathrm{mM}$ Tris $(\mathrm{pH}$ 8.3), $2 \mathrm{mM} \mathrm{MgCl}_{2}, 0.1 \mathrm{mg} / \mathrm{ml}$ gelatin, $125 \mu \mathrm{M}$ deoxynucleoside triphosphate (dNTPs) (LAROVA Bio Chemie, EGmb H Germany), 0.5 units of Hotstartaq ${ }^{\mathrm{TM}}$ polymerase (QIAGEN, No 203205) in the PCR buffer (QIAGEN, No 203205 ) and $125 \mathrm{mM}$ of each oligonucleotide primer and then overlaid by $50 \mu$ l of mineral oil (SIGMA, M-5904) (Snounou et al. 1993). The profile for outer PCR (nest I)was 30 cycles as follows: $95^{\circ} \mathrm{C}, 5 \mathrm{~min} ; 58^{\circ} \mathrm{C}, 2 \mathrm{~min} ; 72$ ${ }^{\circ} \mathrm{C}, 2 \mathrm{~min} ; 94^{\circ} \mathrm{C}, 1 \mathrm{~min}$ followed by 1 cycle of $58^{\circ} \mathrm{C}$ for $2 \mathrm{~min}$ and $72{ }^{\circ} \mathrm{C}$ for $5 \mathrm{~min}$. The profile for inner PCR (nest II) was 35 cycle of $95{ }^{\circ} \mathrm{C}, 5 \mathrm{~min} ; 61^{\circ} \mathrm{C}, 2 \mathrm{~min} ; 72^{\circ} \mathrm{C}$, $2 \mathrm{~min} ; 94^{\circ} \mathrm{C}$, $1 \mathrm{~min}$ followed by one cycle of $61{ }^{\circ} \mathrm{C}$ for $2 \mathrm{~min}$ and $72{ }^{\circ} \mathrm{C}$ for $5 \mathrm{~min}$ (Snounou et al. 1993).

\section{Detection of $I L 4$ variable number of tandem repeat (vntr) polymorphism}

The IL4 polymorphism (vntr) (OMIM Number147780) was used in the present study. IL4vntr primer pairs had been used (5'-TAGGCTGAAAGGGGGAAAGC-3'), (5'-CTGTTC ACCTCAACTGCTCC-3') (Mout et al. 1991). The total volume of the reaction was $20 \mu \mathrm{l}$. The PCR reaction was carried out in $50 \mathrm{mM} \mathrm{KCl}, 10 \mathrm{Mm}$ Tris ( $\mathrm{pH} 8.3$ ), $2 \mathrm{Mm}$ $\mathrm{MgCl}_{2}, 250 \mu \mathrm{M}$ deoxynucleoside triphosphate (dNTPs) (LAROVA Bio Chemie, EGmb H Germany), 0.5 units of Hotstartaq $^{\text {TM }}$ polymerase (QIAGEN, No 203205) in the PCR buffer (QIAGEN, No 203205) and $1.0 \mu \mathrm{M}$ of each oligonucleotide primer. The profile for PCR was $94{ }^{\circ} \mathrm{C}$ 5 min 1 cycle, $95{ }^{\circ} \mathrm{C}, 30 \mathrm{~s} ; 60{ }^{\circ} \mathrm{C} 42 \mathrm{~s} ; 72{ }^{\circ} \mathrm{C} 42 \mathrm{~s}$ for 30 cycles. The PCR product size is $183 \mathrm{bp}, 253 \mathrm{bp}$ and $113 \mathrm{bp}$. Negative control samples were included in each reaction. Electrophoresis was performed on $10 \mu$ of the amplification product on $2.5 \%$ MetaPor ${ }^{\circledR}$ agarose gel stained with $3 \mu \mathrm{l}$ ethidium bromide (SIGMA E-1510) and was run for 2-3 h in $1 \mathrm{X}$ TBE buffer (1 M Tris, $50 \mathrm{M}$ EDTA, $1 \mathrm{M}$ Boric acid) at $80 \mathrm{~V}$ and photographed under a standard UV Transilluminator. The DNA fragment sizes were determined using a standard size marker 50 bp DNA Ladder (Gene Ruler ${ }^{\mathrm{TM}}$ ).

\section{Detection of anti-MSP1 P.falciparum IgG, IgG1 and IgG3 using ELISA}

In agreement with previous studies, antibody responses were predominantly IgG1 and IgG3 and there was little IgG2 and IgG4 reactivity. Therefore, analyses were restricted to $\mathrm{IgG1}$ and $\mathrm{IgG} 3$.

The blood filter papers were first incubated for $1 \mathrm{hr}$ at $56{ }^{\circ} \mathrm{C}$. Then were transferred into a $1.5 \mathrm{ml}$ tube; containing $500 \mu \mathrm{l}$ of PBS $(0.05 \%$ Tween and $0.5 \%$ BSA) and incubated for $2 \mathrm{~h}$ at room temperature on a shaker. The samples were then vortexed for a few seconds; and stored at $-20{ }^{\circ} \mathrm{C}$ (Evengard et al. 1988).

The antigen used for this study to detect the $\mathrm{IgG}, \mathrm{IgG} 1$ and IgG3 antibodies was MSP-1-19. This antigen is kindly donated by Patrick Corran at London School of Hygiene and Tropical Medicine/London/UK. Specific indirect ELISA assay was modified to measure the reaction of anti$P$.falciparum merozoite surface-specific serum IgG, IgG1 and IgG3 (Perlmann et al. 1989) isolated from filter papers samples. Spectrophotometric reading of the plates was done at $492 \mathrm{~nm}$.

The quantification of $\operatorname{IgG}, \operatorname{IgG} 1$ and $\operatorname{IgG} 3$ antibody levels employed a method of assigning an arbitrary number

Table 1 Species-specific oligonuclectide primer pair for P.falciparum detection (Snounou et al. 1993)

\begin{tabular}{ll}
\hline Primers & Sequence $5^{\prime}-3^{\prime}$ \\
\hline rPLU5 & 5-CCT GTT GTT GCC TTA AAC TTC-3 \\
rPLU2 & 5-TTA AAA TTG TTG CAG TTA AAA CG-3 \\
rFAL1 & TTA AAC TGG TTT GGG AAA ACC AAA TAT ATT-3 \\
rFAL2 & 5-ACA CAA TGA ACT CAA TCA TGA CTA CCC GTC-3 \\
\hline
\end{tabular}


of units at each dilution of the subsequent standard curve to be determined. Graphs were plotted of dilution by OD readings. The standard curve dilution OD values were plotted against the number of units for each dilution on a $\log / \log$ scale. The slope and intercept of the straight line were then calculated.

A final estimation of the number of units present in each sample was then determined by taking the mean for the number of units from serial dilutions. The whole process of converting sample OD to the mean of units per sample was performed on a Microsoft Excel spreadsheet.

\section{Statistical analysis}

Data entry was performed using Excel program and analyzed using the software Statistical Package for Social Sciences version 20 (SPSS, Inc., Chicago, IL, USA). Differences were considered statistically significant if the $95 \%$ confidence interval was not overlapping or $p$ value $\leq 0.05$. Descriptive statistics, frequency tables and cross tabulation were generated. A Normality test was done for continuous variables, and if found to be a non-normally distributed, log transform was used in the analyses. Means' differences were evaluated by independent T-test and one way ANOVA according to the number of categories of the independent variables. Median and inter-quartile range (IQR) were used for non-normally distributed variables. Genotypic distribution and allelic frequencies of the Il4 vntr polymorphism in all samples were generated. Chisquare $\left(\chi^{2}\right)$, Odd Ratios (OR) tests with $95 \%$ Confidence Interval (CI) and Fisher-Exact test were also performed to detect variations between cases and control and among different clinical manifestation. Data analysis was performed using IBM SPSS version 20 IBM (IBM Corp. Released 2011), genetics package (Gregory et al. 2019), ggplot2 package (Wickham 2009) on R statistical environment version 3 ( $\mathrm{R}$ core team 2016).

\section{Results}

The study participants were categorized into four age groups: group $1(<10$ years), group 2 (11-20 years), group 3 (21-30 years) and group 4 ( $\geq 30$ years).

Males represented $63(52.5 \%)$ of the study participants while 57(47.5\%) were females. The general clinical appearance of the study participants was found to be 58 (48.3\%) ill, 12 (10\%) severely ill and 50 (41.6\%) were well. No antimalarial prophylaxis was used by the study participants.

\section{Detection of the parasite density}

Seventy one $(59.1 \%)$ out of 120 study participants who attended the hospital clinics were found infected with $P$. falciparum. All participants enrolled as a control group was negative at the thick-smear examination for $P$. falciparum. Species specific primers have produced a characteristic P.falciparum size band of 205 bp using PCR.

Malaria parasitaemia ranged from 1000 to 16,000 parasite per $\mu$ blood. The parasitaemia levels showed a nonnormal distribution with a median of 8000 parasites $/ \mu 1$ blood and IQR (1525-12,000 parasites/ $\mu$ l). A statistically significant difference in parasitaemia levels was observed between different age groups using Kruskal Wallis Test $(p$ value $=0.024)$. Age group $1(<10$ years $)$ was shown the highest mean level of parasitaemia (median $=12,000$ parasite/ $\mu \mathrm{l}$ blood). This is followed by age group 21-30 years $($ median $=10,000.0)$ and 31 years or more (median $=8000.0)$ and the lowest was the age group 11-20 years $($ median $=1600.0) \quad$ parasite $/ \mu \mathrm{l}$ of blood (Fig. 1). A total of 40 (56.3\%) of the P. falciparum malaria positive samples were males while 31 (43.6\%) were females.

\section{Allele frequency of human IL4vntr polymorphisms among study participants}

There were three genotypic combinations of human IL4vntr polymorphisms observed in the study. Allele 1 was represented by a 183 bp fragment, whereas allele 2 was represented by a $253 \mathrm{bp}$ fragment. Allele3 (113 bp) was not detected among study participants. The genotypic distribution and allelic frequencies were shown in Tables 2 and 3 .

There was no significant difference in the distribution of both genotypic and allelic frequencies between cases and control group using chi square test. Similarly, there was no significant difference in the distribution of both genotypic and allelic frequencies as among different clinical manifestation using Fisher-Exact test $(p>0.05)$. Fisher exact test was used to compare the heterozygous frequency and the homozygous due to the small number of heterozygous.

\section{Variation of anti-P.falciparumMSP1-19 IgG, IgG1 and IgG3 levels with parasitaemia}

The mean for total anti-P.fMSP1-19 IgG level was $8794.79 \pm 423.7 \mathrm{IU} / \mathrm{ml}$ with a median of (9075.26) and that for anti-P.fMSP1-19 IgG1 level was $5684.91 \pm 927.24 \mathrm{IU} / \mathrm{ml}$ with a median of (4989.34) and the mean of anti-P.fMSP1-19 IgG3 was $5575.44 \mathrm{IU} / \mathrm{ml} \pm$ $1019.49 \mathrm{IU} / \mathrm{ml}$ with a median of (4919.06). 
Fig. 1 Variation of parasitaemia median with age groups. The box represents the interquartile range of parasitaemia levels within each age group. The horizontal line within the box is the median of parasirtaemia level. The spikes mark the lowest and highest quartile

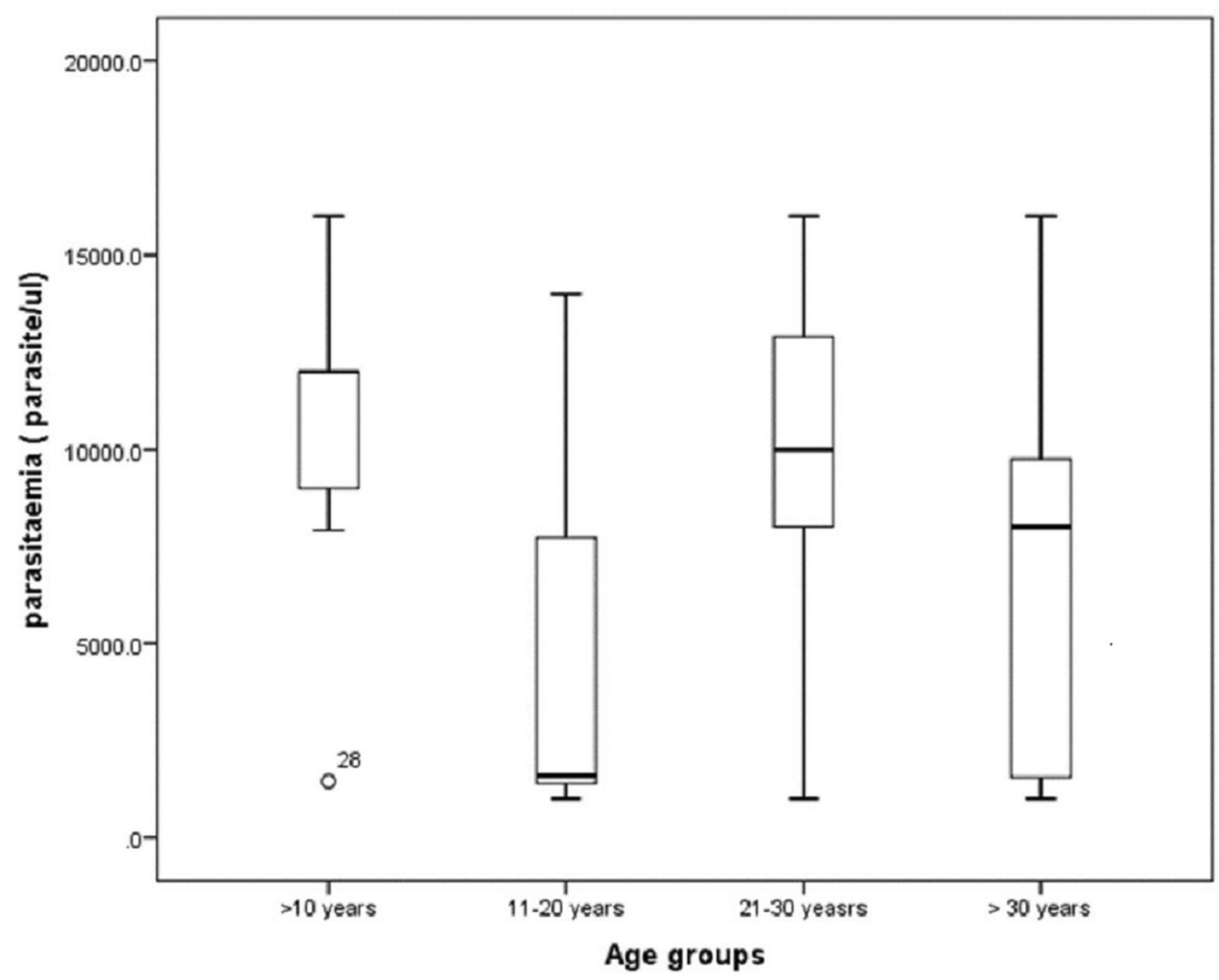

Table 2 The distribution of the genotypes between the cases and control

\begin{tabular}{llllll}
\hline & & Cases $\mathrm{n}=70(\%)$ & Controls $\mathrm{n}=50(\%)$ & Chi square $(\mathrm{p}$ value $)$ & OR $(\mathrm{CI}=95)$ \\
\hline Genotypes & 183 & $37(53 \%)$ & $28(55 \%)$ & $0.116(0.733)$ & $1.135(0.547-2.354)$ \\
& $183 / 253$ & $7(10 \%)$ & $2(4 \%)$ & $1.514(0.219)$ & $0.375(0.075-1.887)$ \\
Alleles & 253 & $26(37 \%)$ & $20(41 \%)$ & $0.101(0.751)$ & $1.128(0.536-2.377)$ \\
& 183 & $81(58 \%)$ & $56(57 \%)$ & $0.101(0.751)$ & $1.128(0.536-2.377)$ \\
& 253 & $59(42 \%)$ & $42(43 \%)$ & & $1.135) 0.547-2.354)$ \\
\hline
\end{tabular}

$p$ value consisdered significant if $<0.05$

Table 3 The distribution of the genotypes among the different clinical manifestations

\begin{tabular}{llllll}
\hline & & Ill $\mathrm{n}=58(\%)$ & Severely ill $\mathrm{n}=12(\%)$ & Well $\mathrm{n}=50(\%)$ & Fisher exact $(\mathrm{p}$ value $)$ \\
\hline Genotypes & 183 & $31(53 \%)$ & $6(50 \%)$ & $28(55 \%)$ & $1.833(0.775)$ \\
& $183 / 253$ & $6(10 \%)$ & $1(8 \%)$ & $2(4 \%)$ & $20(41 \%)$ \\
Alleles & 253 & $21(36 \%)$ & $5(42 \%)$ & $56(57 \%)$ & $0.365(0.856)$ \\
& 183 & $68(59 \%)$ & $13(54 \%)$ & $42(43 \%)$ & \\
\hline
\end{tabular}

$p$ value consisdered significant if $<0.05$

Using Kruskal-Wallis rank sum test, anti-P.fMSPI-19 $\operatorname{Ig} G$ levels showed significant differences among age groups $(p<0.001)$ with group 3 (21-30 years) showing the highest levels of anti-P.fMSP1-19 IgG (Fig. 2). While there was no significant difference between anti-P.fMSP119 IgG1, anti-P.fMSP1-19 IgG3 and age groups (the $p$ values were 0.5 and 0.08 respectively).
Parasitaemia had shown a significant negative correlation with age indicated by spearman correlation $(\mathrm{r}=-0.262$ and $p$ value $=0.043)$ and with anti-P.fMSP 1 19 IgG1 $(\mathrm{r}=-0.418, p$ value $=0.047)$. This a borderline significant and this may be due to high variation in parasitemia between patients. However, it was positively correlated with anti-P.fMSPI-19 IgG3 and anti-P.fMSP1-19 
Fig. 2 Variation of antiP.fMSP1-19 IgG median with age groups. The box represents the interquartile range of antiP.fMSP1-19 IgG levels within each age group. The horizontal line within the box is the median of anti-P.fMSP1-19 IgG level. The spikes mark the lowest and highest quartile

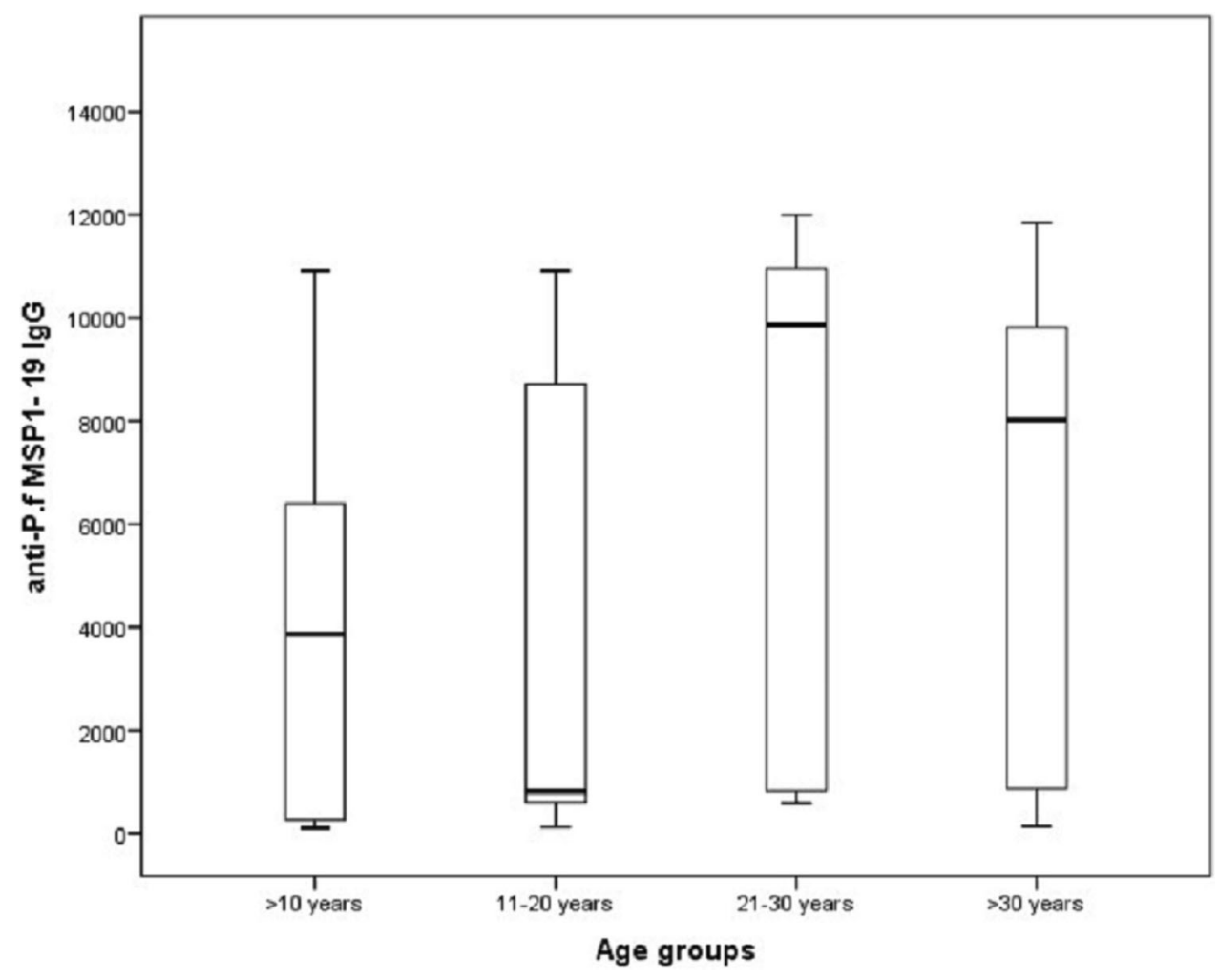

IgG but these correlations were found to be statistically not significant. Correlations and simple linear regression were illustrated in Figs. 3, 4 and Table 4 which is representing spearman correlation coefficients and $p$ values.

Only anti-P.fMSP1-19 IgG showed a significant association with general appearance using Kruskal Wallis ( $p$ value $<0.001$ ). That implicates that variations in $\mathrm{IgG}$ have a significant effect on the variations on the general appearance. Anti-P.fMSP1-19 IgG has observed to be increased in ill and severely ill study participants compared with control as shown in Fig. 5.

No significant associations were obtained between antiP.fMSP1-19 levels with IL4vntr genotypes.

The median for total anti-P.fMSP1-19 IgG1 and IgG3 levels was lower in males compared to females. The variation in IgG3 levels was statistically significant ( $p$ value $<0.001$ ) while it's not significant for the levels of anti-P.fMSP1-19 IgG1 ( $p$ value $=0.129)$. The median level of total anti-P.fMSP1-19 IgG was higher in males compared to females but this difference was not statistically significant $(p$ value $=0.346)($ Fig. 6) .

\section{Discussion}

We aimed in this study to investigate the possible association of clinical, parasitological and immunological phenotypes of Plasmodium falciparum using mainly parasitaemia, IL4vntr polymorphism and anti-P.f-MSP1-19 IgG, IgG1 and IgG3 levels as markers.

Males represented $54.1 \%$ of the study participants while $45.9 \%$ were females. This might indicate that males are more exposed to malaria infection compared to females and/or they might be males have access to hospitals more than females. This is in agreement with a previous study in eastern Sudan (Allam et al. 2008).

The general clinical appearance of the study participants was found to be $58(48.3 \%)$ mild or moderately ill and 12 (10\%) were severely ill. The general appearance of malaria cases in central Sudan is usually observed to be uncomplicated malaria rather than severe malaria (Ali et al. 2011).

In the present study, we observed a significant variation of parasitaemia with age, with age group 1 (0-10 years) showed the highest values of parasitaemia. This is in agreement with previous observations who showed that children below 5 years of age had high parasitaemia while low parasitaemia levels were found in older age groups (Houngbedji et al. 2015). In high endemicity areas, the early exposition to mosquito bites builds up a partial immunity, which in turn results in lower levels of $P$. falciparum infection and parasitaemia with age (Sharma et al. 2004).

Measurements of the anti-P.fMSP1-19 IgG, IgG1 and IgG3 response by ELISA has shown relatively high geometric mean. Using Kruskal-Wallis rank sum test, anti- 
Fig. 3 Scatterplot with regression line representing the correlation of parasitaemia with age

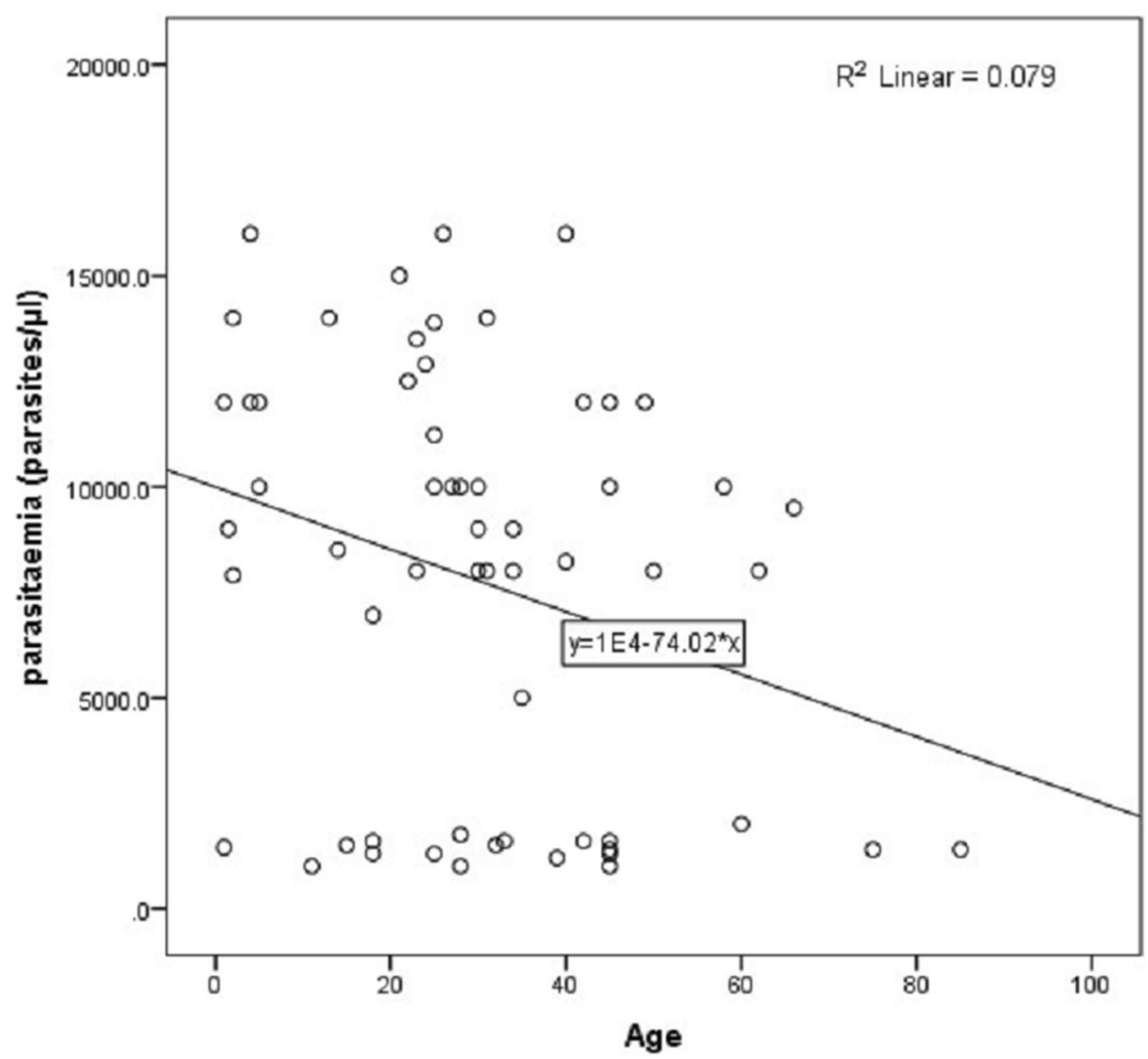

P.fMSP1-19 IgG levels showed significant differences with age groups $(p<0.001)$ with group 3 (21-30 years) showing the highest levels followed by group 4 ( $>30$ years). Adults have shown the highest levels. In a previous study, IgG was higher in infants than children which could be explained by the presence of maternal antibodies, due to the differential placental transfer (Palmeira et al. 2012). A recent study in Cameron has reported an increase in IgG and IgG subclasses levels in an agedependent manner in different study sites except in low transmission settings known as sudano-sahelian strata (Kwenti et al. 2019), which resembles to a great extent the strata of malaria in Khartoum state. An accumulation of anti-PfMSP1-19 IgG in adults may suggest the development of age-dependent immunity. However, in our study, this does not seem to help in protection from the risk of high parasitaemia as a positive correlation was observed between anti-PfMSP1-19 IgG and parasitaemia levels in adults and in infants age group 1 (0-10 years). Moreover, the correlation we have observed also simply could reflect the higher level of exposure experienced by those children who are semi-immune, and the truly protective components of acquired immunity may be elicited by other antigens or factors we have not examined. A significant negative correlation of anti-Pf MSP1-19 IgG1 with parasitaemia was observed in this study and this might indicate a protective role for IgG1. This is in agreement with a previous study that showed malaria protective immunity is mostly associated with the cytophilic subclasses IgG1 and IgG3 (Adamou et al. 2019; Al-Yaman et al. 1996). Although antibodies to MSP1-19 have been shown to be associated with both exposure and protection from disease, the fine specificities of such responses may contribute to protection (Okech et al. 2004; Lusingu et al. 2005; Tongren et al. 2006).

The IgG level to MSP1-19 has shown to be increased in ill and severely ill study participants compared to well or control subjects. The study in Cameron has reported the increase of total $\mathrm{IgG}$ in children positive for clinical malaria compared to negative children (Kwenti et al. 2019). A previous study in West Africa showed that the immune response to MSP1-19 needs a rate of exposure to be build-up where it is affected by low prevalence. On the other hand, elevated concentration of antimalarial IgG antibodies may reflect not only recent exposure but also previous exposure to parasites and therefore might not necessarily correlate with protection (Al-Yaman et al. 1996). 
Fig. 4 Correlation and regression line of parasitaemia with anti-P.fMSP1-19 IgG1

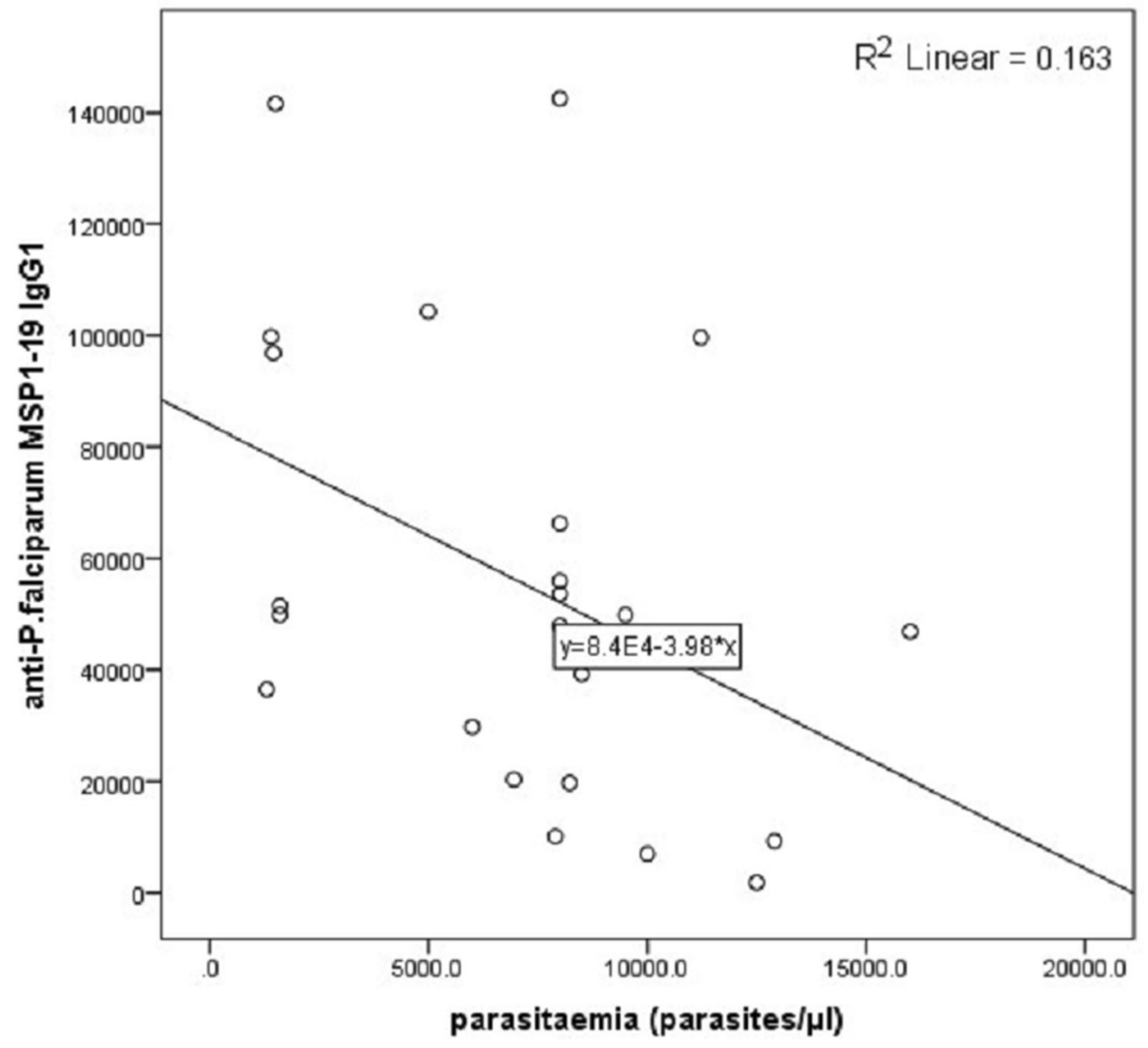

Table 4 Correlation of parasitaemia with anti-Pf-MSP1-19 IgG, IgG1, IgG3 levels and age among study participants

\begin{tabular}{llcclc}
\hline & & parasitaemia & Anti-P.fMSP1-19 IgG & Anti-P.fMSP1-19 IgG1 & Anti-P.fMSP1-19 IgG3 \\
\hline Anti-P.fMSP1-19 IgG & $\mathrm{R}$ & 0.046 & & & \\
& $p$ value & 0.704 & & \\
Anti-P.fMSP1-19 IgG1 & $\mathrm{R}$ & $-.418^{*}$ & -0.04 & & \\
& $p$ value & 0.047 & 0.833 & 0.127 & 0.553 \\
Anti-P.fMSP1-19 IgG3 & $\mathrm{R}$ & -0.062 & -0.277 & 0.209 & -0.274 \\
& $p$ value & 0.755 & 0.131 & 0.277 & 0.15 \\
\hline
\end{tabular}

$p$ value consisdered significant if $<0.05$

The mean for total anti-P.fMSP1-19 IgG1 and IgG3 level were lower in males compared to females, The difference for IgG3 levels was statistically significant ( $p$ value $<0.001$ ). Although the majority of studies have reported no significant variation of IgG and IgG subclasses with sex, only one previous study in Africa has supported our findings and showed a significant increase in levels of IgG3 against P.f-MSP1-19 in females compared to males (Dobaño et al. 2019).
We had also investigated in this study the frequency of human ILAvntr alleles. There was no significant difference in the distribution of both genotypic and allelic frequencies between cases and control group as well as among different clinical manifestation. Allele 3 (113 bp) was not detected among the study participants. A previous study has indicated the association of ILAvntr allele 1 (183 bp) with cerebral malaria but not with severe malaria anemia or uncomplicated malaria (Gyan et al. 2004). There is a need 
Fig. 5 Boxplot representing the increase of anti P.f-MSP1-19 $\mathrm{IgG}$ median with the disease severity. Horizontal bars indicate the median antibody levels, MSP1 was used as antigen; the box represents the interquartile range of antiP.fMSP1-19 IgG levels within each class. The horizontal line within the box is the median of anti-P.fMSP1-19 IgG level. The spikes mark the lowest and highest quartile. MSP1-19 antigen was used
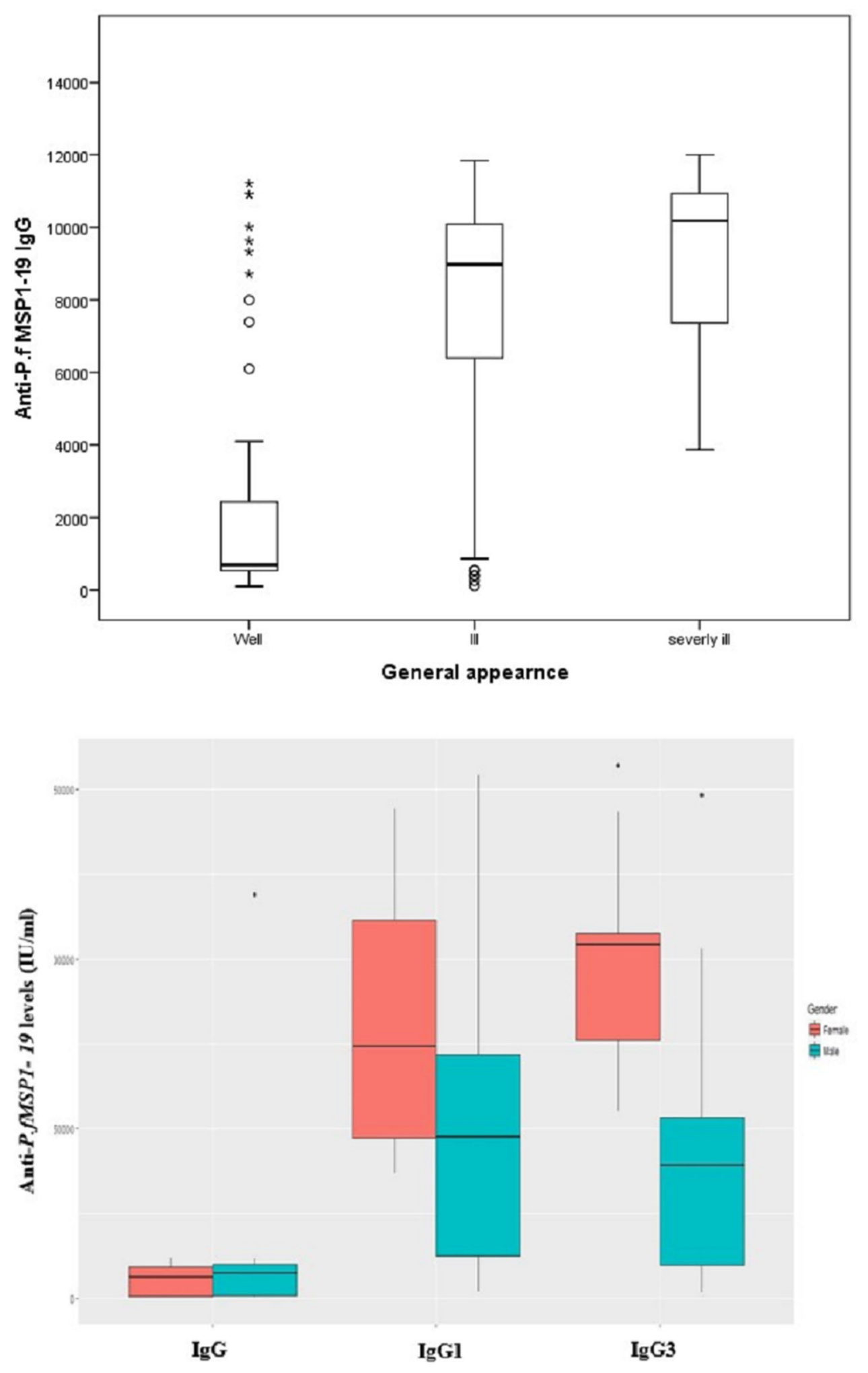

Fig. 6 Variation of antiP.fMSP1-19 IgG, IGg1 and $\mathrm{IgG} 3$ between males and females Variation of antiP.fMSP1-19 IgG, IgG1 and IgG3 medians between males and females Horizontal bars indicate the median antibody levels, MSP1 was used as antigen; the box represents the interquartile range of antiP.fMSP1-19 antibody levels within each class. The horizontal line within the box is the median of anti-P.fMSP1-19 antibody level. The spikes mark the lowest and highest quartile. MSP1-19 antigen was used. for further investigation and more sampling to find out if the study participants who have IL4vntr allele 1 are more prone to get infected by malaria and how are this allele correlates to the physiological changes in malaria patients.
The ILAvntr alleles may constitute a factor that protects against $P$. falciparum malaria, or be associated with such a factor (Gyan et al. 2004). The ability to assess the role of ILA gene in $P$. falciparum malaria infection inheritance is 
largely dependent on estimates of trait heritability. This might need a family-based study rather than cross-sectional study to detect heritability. Traits of the highest heritability probably show a greater chance for detecting regions or markers that contribute to that trait. Ideally, marker genes should be close to the disease-causing gene and highly polymorphic (Morton 1998; Morton and Collins 1998). Other factors such as sampling procedures and analytical methods that are used to locate the markers are also of significant importance. Our previous studies have shown and highlighted the significant linkage and allelic association of these regions with phenotypes related to $P$. falciparum infection in Bantu clan in Nyamisati village eastern Tanzania (Carpenter et al. 2007, 2009).

Unlike previous studies, we were not able to detect an association between ILAvntr polymorphism and the levels of anti-P.fMSP1-19 antibodies among study participants which mightily due to limited sample size. Variation in ILAvntr alleles has been shown previously to be associated with increased levels of IgG among Fulani ethnic group in West Africa (Luoni et al. 2001). Human epidemiological data and murine experimental models indicate that IL-4 plays a key role in the induction of specific antibodies during blood stages of malaria (Perkmann et al. 2005; Troye-Blomberg et al. 1999). However, its role as a determinant of clinical outcomes remains uncertain. The reason for the insignificant association is probably due to: first, the polymorphism itself may not be functional but it acts as a genetic marker of other functional polymorphisms; second, the polymorphisms may directly regulate IL4 production only in the presence of other genetic determinants.

\section{Conclusion and recommendation}

Malaria infection is affected by many factors like differences in immunity related to variation in malaria exposure, difference in parasite virulence (clones) and genetics of the human host. The understanding of parasite and host dynamics in course of malaria infection will help to develop effective chemotherapy and successful vaccine protection. The development of parasite resistance to drugs and of vector's resistance to insecticides, as well as the lack of an effective vaccine, emphasizes the need for alternative weapons to fight the disease.

From the present study, it was concluded that IgG1 levels to MSP1-19 were found to be negatively correlated with parasitaemia and anti-PfMSPI-19 IgG has shown to be increased significantly in ill and severely ill. Further studies are needed to ascertain the role of $\operatorname{IgG}$ and $\operatorname{IgG} 1$ in protection and to investigate the $\mathrm{IgG}$ and subclasses response against other antigenic markers like apical membrane antigen 1 (AMA1). The development of partial immunity in people living in malaria endemic areas is complex. These findings are valuable for advancing vaccine development by providing evidence supporting merozoite antigens as targets of protective immunity in humans, and to help identify antigens that confer protection from malaria. Although, IL4vntr polymorphism showed to be one of the markers that are linked to a protective effect in patients with malaria no significant difference in the distribution of both genotypic and allelic frequencies between cases and control group as well as among different clinical manifestation using was detected in this study. Further studies are recommended to explore the role of ILA gene in protection, and the role of other malaria candidate genes like ILIO and TNF which will help in understanding the susceptibility of $P$. falciparum infection. Improved surveillance data that allows for the analysis of seasonality, age and other risk factors need to be collected to design effective small area interventions as Khartoum state to target malaria elimination.

Acknowledgements This project is partially funded by University of Khartoum and Sudanese Ministry of Higher Education and Scientific Research.

Author contributions HMA conceived, designed the experiments, and wrote the manuscript: IAA, HA, TM performed the experiments: FM, HMA, MMA analyzed the data. MMA, IME contributed reagents, materials, analysis tools and critically revised the manuscript. All authors have revised the manuscript.

\section{Compliance with ethical standards}

Competing interests The authors have declared that no competing interests exist.

\section{References}

A-Elgadir TM, Elbashir MI, Berzins K, Masuadi EM, A-Elbasit IE, ElGhazali G et al (2008) The profile of IgG-antibody response against merozoite surface proteins 1 and 2 in severe Plasmodium falciparum malaria in Eastern Sudan. Parasitol Res 102(3):401-409

Adamou R, Dechavanne C, Sadissou I, d'Almeida T, Bouraima A, Sonon P et al (2019) Plasmodium falciparum merozoite surface antigen-specific cytophilic IgG and control of malaria infection in a Beninese birth cohort. Malar J 18(1):194. https://doi.org/10.1186/s12936-019-2831

Ali AA, Elhassan EM, Magzoub MM, Elbashir MI, Adam I (2011) Hypoglycaemia and severe Plasmodium falciparum malaria among pregnant Sudanese women in an area characterized by unstable malaria transmission. Parasites Vectors 4:88

Al-Yaman F, Genton B, Kramer KJ, Chang SP, Hui GS, Baisor M et al (1996) Assessment of the role of naturally acquired antibody levels to Plasmodium falciparum merozoite surface protein-1 in protecting Papua New Guinean children from malaria morbidity. Am J Trop Med Hyg 54(5):443-448 
Allam MM, Alkadarou TA, Ahmed BG, Elkhair IS, Alansary EH, Ibrahim ME et al (2008) Hyper-reactive Malarial Splenomegaly (HMS) in malaria endemic area in Eastern Sudan. Acta Trop 105(2):196-199

Carpenter D, Abushama H, Bereczky S, Farnert A, Rooth I, TroyeBlomberg $M$ et al (2007) Immunogenetic control of antibody responsiveness in a malaria endemic area. Hum Immunol 68(3):165-169

Carpenter D, Rooth I, Farnert A, Abushama H, Quinnell RJ, Shaw MA (2009) Genetics of susceptibility to malaria related phenotypes. Infect Genet Evol 9(1):97-103

Dobaño C, Santano R, Vidal M, Jiménez A, Jairoce C, Ubillos I, Dosoo D, Aguilar R et al (2019) Differential patterns of IgG subclass responses to Plasmodium falciparum antigens in relation to malaria protection and RTS, S vaccination. Front Immunol 10:439

Dodoo D, Theander TG, Kurtzhals JA, Koram K, Riley E, Akanmori BD et al (1999) Levels of antibody to conserved parts of Plasmodium falciparum merozoite surface protein 1 in Ghanaian children are not associated with protection from clinical malaria. Infect Immun 67(5):2131-2137

Evengard B, Linder E, Lundbergh P (1988) Standardization of a filterpaper technique for blood sampling. Ann Trop Med Parasitol 82(3):295-303

Federal Ministry of Health, Annual statistical reports Federal Ministry of Health (2000-2016), Khartoum 2000-2016, Ministry of Health, Khartoum, Sudan

Fowkes FJ, Richards JS, Simpson JA, Beeson JG (2010) The relationship between anti-merozoite antibodies and incidence of Plasmodium falciparum malaria: a systematic review and metaanalysis. PLoS Med 7(1):e1000218

Giha HA, Nasr A, Iriemenam NC, Balogun HA, Arnot D, Theander TG et al (2010) Age-dependent association between $\mathrm{IgG} 2$ and IgG3 subclasses to Pf332-C231 antigen and protection from malaria, and induction of protective antibodies by sub-patent malaria infections, in Daraweesh. Vaccine 28(7):1732-1739

Gilles HM (1993) Epidemiology of malaria. In: Gilles HMWD (ed) Bruce-Chwatt's essential malariology, 3rd edn. Edward Arnold, London, pp 124-164

Gregory W, Gregor G, Leisch F, Man M (2019) Genetics: population genetics. $\mathrm{R}$ package version 1.3.8.1.1. https://cran.r-project.org/package=genetics

Gyan BA, Goka B, Cvetkovic JT, Kurtzhals JL, Adabayeri V, Perlmann $\mathrm{H}$ et al (2004) Allelic polymorphisms in the repeat and promoter regions of the interleukin- 4 gene and malaria severity in Ghanaian children. Clin Exp Immunol 138(1):145-150

Houngbedji CA, N'Dri PB, Hurlimann E, Yapi RB, Silue KD, Soro G et al (2015) Disparities of Plasmodium falciparum infection, malaria-related morbidity and access to malaria prevention and treatment among school-aged children: a national cross-sectional survey in Cote d'Ivoire. Malar J 14:7

Iriemenam NC, Khirelsied AH, Nasr A, ElGhazali G, Giha HA, Elhassan AETM et al (2009) Antibody responses to a panel of Plasmodium falciparum malaria blood-stage antigens in relation to clinical disease outcome in Sudan. Vaccine 27(1):62-71

Kwenti TE, Kukwah TA, Kwenti TDB et al (2019) Comparative analysis of $\mathrm{IgG}$ and $\mathrm{IgG}$ subclasses against Plasmodium falciparum MSP-119 in children from five contrasting bioecological zones of Cameroon. Malar J 18:16

Lokossou AG, Dechavanne C, Bouraima A, Courtin D, Le Port A, Ladekpo R et al (2013) Association of IL-4 and IL-10 maternal haplotypes with immune responses to $P$. falciparum in mothers and newborns. BMC Infect Dis 13:215

Luoni G, Verra F, Arca B, Sirima BS, Troye-Blomberg M, Coluzzi M et al (2001) Antimalarial antibody levels and IL4 polymorphism in the Fulani of West Africa. Genes Immun 2(7):411-414
Lusingu JP, Vestergaard LS, Alifrangis M, Mmbando BP, Theisen M, Kitua AY et al (2005) Cytophilic antibodies to Plasmodium falciparum glutamate rich protein are associated with malaria protection in an area of holoendemic transmission. Malar J 4:48

Malaria Control Programme (2008) National Insecticid-Treated Nets (ITNs) strategy. Ministry of Health, Khartoum

Marsh K (1992) Malaria-a neglected disease? Parasitology 104(Suppl):S53-69

Morton NE (1998) Significance levels in complex inheritance. Am J Hum Genet 62(3):690-697

Morton NE, Collins A (1998) Tests and estimates of allelic association in complex inheritance. Proc Natl Acad Sci U S A 95(19):11389-11393

Mout R, Willemze R, Landegent JE (1991) Repeat polymorphisms in the interleukin-4 gene (IL4). Nucleic Acids Res 19(13):3763

Nguer CM, Diallo TO, Diouf A, Tall A, Dieye A, Perraut R et al (1997) Plasmodium falciparum- and merozoite surface protein 1-specific antibody isotype balance in immune Senegalese adults. Infect Immun 65(11):4873-4876

Okech BA, Corran PH, Todd J, Joynson-Hicks A, Uthaipibull C, Egwang TG et al (2004) Fine specificity of serum antibodies to Plasmodium falciparum merozoite surface protein, PfMSP1(19), predicts protection from malaria infection and highdensity parasitemia. Infect Immun 72(3):1557-1567

Palmeira P, Quinello C, Silveira-Lessa AL, Zago CA, CarneiroSampaio M (2012) IgG placental transfer in healthy and pathological pregnancies. Clin Dev Immunol 2012:985646

Perkmann T, Winkler H, Graninger W, Kremsner PG, Winkler S (2005) Circulating levels of the interleukin (IL)- 4 receptor and of IL-18 in patients with Plasmodium falciparum malaria. Cytokine 29(4):153-158

Perlmann P, Troye-Blomberg M (2002) Malaria and the immune system in humans. Chem Immunol 80:229-242

Perlmann H, Perlmann P, Berzins K, Wahlin B, Troye-Blomberg M, Hagstedt $M$ et al (1989) Dissection of the human antibody response to the malaria antigen Pf155/RESA into epitope specific components. Immunol Rev 112:115-132

Plowe CV, Cortese JF, Djimde A, Nwanyanwu OC, Watkins WM, Winstanley PA et al (1997) Mutations in Plasmodium falciparum dihydrofolate reductase and dihydropteroate synthase and epidemiologic patterns of pyrimethamine-sulfadoxine use and resistance. J Infect Dis 176(6):1590-1596

R Core Team (2016) R: a language and environment for statistical computing. R Foundation for Statistical Computing, Vienna, Austria. https://www.R-project.org/

Sharma SK, Chattopadhyay R, Chakrabarti K, Pati SS, Srivastava VK, Tyagi PK, Mahanty S, Misra SK, Adak T, Das BS, Chitni SE (2004) Epidemiology of malaria transmission and development of natural immunity in a malaria-endemic village, San Dulakudar, in Orissa state, India. Am J Trop Med Hyg 71:457-465

Snounou G, Viriyakosol S, Zhu XP, Jarra W, Pinheiro L, do Rosario VE et al (1993) High sensitivity of detection of human malaria parasites by the use of nested polymerase chain reaction. Mol Biochem Parasitol 61(2):315-320

Tongren JE, Drakeley CJ, McDonald SL, Reyburn HG, Manjurano A, Nkya WM et al (2006) Target antigen, age, and duration of antigen exposure independently regulate immunoglobulin $G$ subclass switching in malaria. Infect Immun 74(1):257-264

Troye-Blomberg M, Worku S, Tangteerawatana P, Jamshaid R, Soderstrom K, Elghazali G et al (1999) Human gamma delta T cells that inhibit the in vitro growth of the asexual blood stages of the Plasmodium falciparum parasite express cytolytic and proinflammatory molecules. Scand J Immunol 50(6):642-650

Wickham H (2009) ggplot2: elegant graphics for data analysis. Springer, New York 
World Malaria Report (2018) World Health Organisation. https://apps.who.int/iris/bitstream/handle/10665/275867/ 9789241565653-eng.pdf?ua=1
Publisher's Note Springer Nature remains neutral with regard to jurisdictional claims in published maps and institutional affiliations. 